\title{
The spatial and temporal relationship between oxidative stress and neuronal degeneration in 3-nitropropionic acid model
}

\author{
Thierry Delorme, Mohammad Najafi, Payman Nasr* \\ Department of Biological Sciences, Kent State University, Ashtabula, USA \\ Email: *"pnasr@kent.edu
}

Received 15 June 2012; revised 29 July 2012; accepted 14 August 2012

\begin{abstract}
The current study investigates the role of oxidative stress and calcium homeostasis in the development of selective striatal lesions in metabolic impairment model caused by 3-nitropropionic acid (3NP). In this report, we examined the distribution of oxidative stress markers and the production of mitochondrial reactive oxygen species in the presence of 3NP in male Sprague-Dawley rats. Protein oxidation was assessed using 3-nitrotyrosine immunoreactivity, while DNA oxidative damage was evaluated by poly (ADP-ribose) polymerase-1 activity. The Reactive Oxygen Species (ROS) production was determined in isolated mitochondrial from striatum and cerebellum of two age groups following 3NP and variable calcium concentration. The results demonstrate that increased 3nitro-tyrosine level is the most robust in the striatum and the least evident in the cerebellum following 4 days of 3NP treatment. No significant change in the levels of poly ADP-ribosylated proteins was observed, likely due to a rapid PARP-1 cleavage as detected by the appearance of $50 \mathrm{kDa}$ necrotic fragment. In mitochondrial isolates, there was no immediate increase in mitochondrial ROS following 3NP in either striatum or cerebellum; however, calcium addition resulted in a concentration dependent increase in reactive oxygen species in striatal mitochondria of the older animals. These results suggest that in aging, mitochondria become more susceptible to the generation of ROS in conditions that cause a concurrent compromised in mitochondrial calcium concentration. This finding implicates mitochondria dysfunction as a key cellular target in pathological states that are associated with metabolic impairment. The results also reinforce the notion that mitochondrial function in the striatum and cerebellum respond differently to the aging process, which may explain the variable regional vulnerability in 3NP model.
\end{abstract}

${ }^{*}$ Corresponding author.
Keywords: Energy Impairment; 3-Nitropropionic Acid; Oxidative Stress; Neurodegeneration

\section{INTRODUCTION}

Numerous environmental toxins can compromise mitochondrial function and impair energy metabolism. One such toxin is 3-nitropropionic acid (3NP) which is produced by a number of fungal and plant species [1-2]. $3 \mathrm{NP}$ is an irreversible inhibitor of succinate dehydrogenase (SDH), a component of mitochondrial complex II and the Krebs cycle [3]. Severe 3NP poisoning results in motor impairment accompanied with basal nuclei (striatum) degeneration in humans, primates and rodents [4]. Since the discovery of $3 \mathrm{NP}$ as the causative agent in sugarcane poisoning and striatal degeneration, many investigators have utilized this compound to reproduce the cognitive and motor deficits seen in neurodegenerative diseases such as Huntington's disease in animal models [5-8].

There are many reports of 3NP toxicity in humans due to sugar cane infestation by Arthinium species. Over a long period of storage, Arthinium species are capable of producing 3NP. In 1970's and 80's, sugarcane, being a common snack in China, resulted in more than 800 reported cases of $3 \mathrm{NP}$ toxicity and 88 deaths [9]. In mild cases of 3NP toxicity, the patients developed lethargy, gastritis, nausea and vomiting. In severe cases, the symptoms consisted of severe seizures, coma, brain edema and neurodegeneration in caudate putamen, globusp allidus and lenticular nuclei $[10,11]$.

In mammals, systemic administration of $3 \mathrm{NP}$ results in progressive locomotor impairment and selective striatal lesions [12-14]. In rats, the dorsolateral striatum is generally considered homologous to the putamen in humans, a region directly involved in the control of movement, while the ventromedial aspect of the rat striatum is similar to the caudate nucleus [15]. Following systemic $3 \mathrm{NP}$ administration in rats, dorsolateral striatum is the 
main site of neurodegeneration, whereas ventromedial striatum is relatively spared. The majority of the neurons in dorsolateral striatum are medium-sized spiny neurons which receive strong glutamatergic inputs from the cerebral cortex and thalamus, as well as a dopaminergic input from the substantia nigra. In addition, the striatum receives minor inputs from raphe nuclei, the globus pallidus and subthalamic nucleus (for review see [16-18]).

The 3NP model presents a number of technical advantages over other mitochondrial toxins such as malonate. The main advantage of $3 \mathrm{NP}$ is that it can be administered systemically and readily crosses the blood-brain barrier [12]. Moreover, despite the inhibition of SDH throughout the body, 3NP is highly selective in producing striatal lesions similar to those seen in Huntington's disease in which the medium-sized spiny neurons in striatum are the most vulnerable [19]. 3NP also causes more severe neurotoxicity in the older experimental animals compared to the younger ones [20,21]. In addition, $3 \mathrm{NP}$ is relatively inexpensive and simple to utilize. Taken together, $3 \mathrm{NP}$ offers a practical model to study neurodegenerative disorders associated with metabolic impairment.

$3 \mathrm{NP}$ administration results in uniform inhibition of SDH activity throughout the brain [22,23], and the reasons for the selective vulnerability of striatal neurons are not well understood. Growing evidence has implicated secondary excitotoxicity, oxidative stress and calcium homeostasis as key factors in the preferential striatal degeneration in the 3NP model [24-28].

Striatum receives a strong glutamatergic input from the cerebral cortex, which renders the striatum vulnerable to excitotoxicity. In vitro, in the presence of $3 \mathrm{NP}$, N-Methyl-D-Aspartate (NMDA) receptor inhibition shifts the cell death from predominantly necrotic to apoptotic characteristics, but does not prevent the cell death [29]. In vivo, decortication protects the striatum against degeneration following systemic 3NP administration [19].

NMDA receptor activation is also closely linked to oxidative stress via Nitric Oxide Synthase (NOS) activity [30-32]. Nitric oxide is a simple diatomic gas with important physiological functions and is produced by NOS in vivo [33-38]. Activation of NOS results in increased production of nitric oxide, which in turn, in the presence of an oxidative environment, readily reacts with superoxide radicals $\left(\mathrm{O}_{2}^{-}\right)$to form peroxynitrite, a toxic oxidant [39]. Tyrosine residues of proteins are particularly susceptible to nitration by peroxynitrite, resulting in the formation of 3-nitrotyrosine [40]. The level of 3-nitrotyrosine can be detected in vivo and have been utilized as a marker for protein oxidation in biological tissues [39,41]. Moreover, increased levels of 3-nitrotyrosine have previously been reported in a number of pathological conditions including amyotrophic lateral sclerosis and Alzheimer's disease [42-45].
Peroxynitrite and its byproducts can also damage DNA, leading to the activation of the repair enzyme poly (ADP-ribose) polymerase-1 (PARP-1) [46]. PARP-1 cleaves $\mathrm{NAD}^{+}$into nicotinamide and ADP-ribose, with subsequent polymerization of ADP-ribose on a set of specific acceptor proteins, in turn altering their structure and initiating the process of DNA repair. PARP-1 is a highly energy-dependent enzyme, and its excessive activation rapidly depletes cellular $\mathrm{NAD}^{+}$and ATP supply, exacerbating the metabolic crisis inflicted by energy impairment.

Others have demonstrated that excessive calcium uptake by mitochondria stimulates the mitochondrial free radical generation $[47,48]$. In vitro analysis demonstrates that the addition of calcium to mitochondria isolates in the presence of rotenone (complex I inhibitor) and antimycin A (complex III inhibitor) results in significant increase in ROS [49-52]. In addition, Jacquard and colleagues have demonstrated that impaired mitochondrial calcium homeostasis is directly implicated in 3NP-induced toxicity [53]. The neurotoxic effect of $3 \mathrm{NP}$ is reported to be due to an improper sequestration of $\mathrm{Ca}^{2+}$ by mitochondria and consequent calpain proteases activation [53].

In summary, the current report examines the role of oxidative stress and calcium homeostasis in the development of selective striatal lesion in metabolic impairment model caused by 3 NP. Protein oxidation was assessed using 3-nitrotyrosine immunoreactivity, while DNA oxidative damage was evaluated by poly (ADP-ribose) polymerase- 1 activity. The reactive oxygen species production was also determined in isolated mitochondrial from striatum and cerebellum of two age groups following $3 \mathrm{NP}$ and variable calcium concentrations.

\section{METHODOLOGY}

\subsection{Materials}

3NP was purchased from Sigma-Aldrich (St. Louis, MO). Protein levels were measured with Pierce BCA kit (Pierce Chemical Company, Rockford, IL). The chemiluminescent substrate for Western blots, Super signal West Pico, was also from Pierce. The anti-nitrotyrosine mouse monoclonal IgG (clone 1A6 Cat\# 05-233) was purchased from Upstate biotechnology (Lake Placid, NY). The PARP-1 cleavage detection kit (Cat\# SK-003) and monoclonal anti-poly (ADP-ribose) antibody against ADPribosylated proteins (Cat\# SA-250) were obtained from BioMol Research Laboratories (Plymouth, PA). All other reagents were from Sigma-Aldrich (St. Louis, MO).

\subsection{Animals}

All experimental protocols involving animals were in accordance with the guidelines published in the NIH 
Guide for the Care and Use of Laboratory Animals and the Society for Neuroscience Guidelines for the Use of Animals in Neuroscience Research. To study the involvement of oxidative stress in 3NP toxicity, SpragueDawley rats were intraperitoneally injected with 3-NP (20 $\mathrm{mg} / \mathrm{kg} /$ day) for $1,2,3$ or 4 days, while the control animals were treated with saline for 4 days. At the end of the treatments, the animals were anesthetized with pentobarbital $(60 \mathrm{mg} / \mathrm{kg})$, decapitated, and the brains were removed immediately. One hemisphere was incubated in $4 \%$ paraformaldehyde for 24 hours at $4^{\circ} \mathrm{C}$ followed by 24 hours incubation in $30 \%$ sucrose in phosphate buffer solution $(\mathrm{pH}$ 7.4) for lesion analysis, and the other hemisphere was dissected and the striatum, hippocampus and cerebellum were removed, frozen on dry ice and stored at $-80^{\circ} \mathrm{C}$ degrees for Western blot analysis

\subsection{Western Blotting}

The tissues of the region of interest (striatum, hippocampus and cerebellum) were homogenized in Trisbuffered saline (50 mM Tris- $\mathrm{HCl}, 150 \mathrm{mM} \mathrm{NaCl}, \mathrm{pH} 7.5)$ containing protease inhibitor (1 mM leupeptin, $25 \mathrm{mM}$ EDTA, $1 \mu \mathrm{M}$ pepstatin A, $200 \mu \mathrm{M}$ AEBSF) then centrifuged at $14,000 \times \mathrm{g}$ for 5 minutes and supernatant was collected. For PARP-1 cleavage products and ADPribosylated proteins, $0.1 \%$ SDS, $1 \%$ Nonidet P-40 and $20 \%$ glycerol were added to the homogenizing buffer. Protein concentrations were determined using the BCA protein Assay, and equal samples were loaded on a gradient SDS-PAGE gel (10 - $40 \mu \mathrm{g} /$ lane). For 3-nitrotyrosine analysis, $10 \mu \mathrm{l}$ of nitrotyrosineimmuno blotting control (Cat\# 05-233, Upstate Biotechnology) was utilized as a positive control, while as a negative control, the primary antibody was excluded in a sister blot. For PARP-cleavage immunoblots, we utilized whole cell extracts of human HL60 leukemia cells containing intact PARP-1 (Cat\# SW101, BioMol Research Laboratories) and whole cell extracts of human HL60 leukemia cells undergoing apoptosis by the chemotherapeutic agent etoposide (Cat\# SW-102, BioMol Research laboratories) as positive controls.

SDS-PAGE was performed according to the method of Laemmli [54] using a mini-gel apparatus (Bio-Rad, Hercules CA). Following SDS-PAGE, polypeptides were transferred electrophoretically onto $0.45 \mu \mathrm{m}$ nitrocellulose membranes. The membranes were blocked for 60 120 minutes in 5\% fat-free milk in TTBS and incubated with the primary antibody in TTBS overnight at room temperature. The membranes were then incubated with peroxidase-conjugated goat anti-mouse or horse antirabbit IgG (Jackson Immunoresearch Laboratories, West Grove, PA) for 60 minutes at room temperature. The blots were developed in SuperSignal West Pico chemiluminescent substrate (Pierce Chemical, Rockford, IL) for 1 minute and exposed to Kodak T-Max X-ray film.

\subsection{Lesion Analysis}

For the fixed tissue, each brain hemisphere was sectioned at $25 \mu \mathrm{m}$ intervals using a cryostat instrument. Every 4th section was mounted on slides and stained with cresyl violet. The sections were imaged using a digital camera, and the total striatal volume and the lesion volume were measured and the percent spared tissue was calculated for each animal. The lesion area was identified by absent or pale cresyl violet staining. The cell loss in the lesion area was confirmed by microscopic examination.

\subsection{Mitochondrial Preparation}

Isolated mitochondria were prepared as previously described with slight modifications [55]. Briefly, SpragueDawley rats $(\mathrm{n}=6$ per group) were anesthetized by sodium pentobarbital $(60 \mathrm{mg} / \mathrm{kg})$, the brains were removed, and the striatum and cerebellum were carefully dissected. All the following steps in mitochondrial isolation were performed at $4^{\circ} \mathrm{C}$. The dissected tissue was minced in ice-cold homogenization buffer $(250 \mathrm{mM}$ sucrose, $20 \mathrm{mM}$ HEPES, 0.1\% BSA, $1 \mathrm{mM}$ EDTA, $\mathrm{pH}$ 7.2) and was rinsed with $10 \mathrm{ml}$ homogenization buffer to remove residual blood. Next, the minced tissue was processed (8 strokes) using a hand-held tissue homogenizer (Thomas Scientific). The resulting homogenate was centrifuged for $3 \mathrm{~min}$ at $1300 \times \mathrm{g}$, the supernatant was removed, centrifuged at $13,000 \times \mathrm{g}$ for $10 \mathrm{~min}$ and the pellet was placed in nitrogen cell bomb and exposed to 1000 psi for 5 minutes to disrupt synaptosomal membranes. The pellets were resuspended in EGTA free isolation buffer and centrifuged at $10,000 \times \mathrm{g}$ for $10 \mathrm{~min}$. Mitochondrial protein concentration was determined using a Pierce BCA kit.

\subsection{ROS Production}

The mitochondrial ROS production assay was performed immediately after mitochondrial isolation. ROS production was measured using the indicator 2', 7'-dichlorofluorescin diacetate (DCF-DA, Molecular Probes, Eugene, OR) as previously described [55,56]. Briefly, $100-150$ $\mu \mathrm{g}$ of isolated mitochondrial protein was incubated in a total volume of $200 \mu \mathrm{l}$ respiration buffer $(215 \mathrm{mM}$ mannitol, $75 \mathrm{mM}$ sucrose, $1 \% \mathrm{BSA}, 2 \mathrm{mM} \mathrm{MgCl}_{2}, 2.5 \mathrm{mM}$ $\mathrm{KH}_{2} \mathrm{PO}_{4}, 20 \mathrm{mM}$ HEPES) at $37^{\circ} \mathrm{C}$ for $15 \mathrm{~min}$ in the presence of $10 \mu \mathrm{M}$ DCF-DA, which was made fresh before each use. The relative amount of mitochondrial free radical generation in the presence of $3 \mathrm{NP}$ with or without calcium $(50 \mu \mathrm{M}-150 \mu \mathrm{M})$ was monitored by measuring changes in fluorescence resulting from DCF-DA oxidation product, DCF, using a CytoFluor 4000 fluorometric plate reader (excitation $490 \mathrm{~nm}$, emission $526 \mathrm{~nm}$ ). 
Addition of $\mathrm{H}_{2} \mathrm{O}_{2}$ was utilized as a positive control and increased DCF fluorescence in a linear style.

\subsection{Statistical Analysis}

For Western blot quantitative analysis, comparisons among groups were made by two-way ANOVA followed by Fisher's PLSD $t$-test. The percent spared striatal tissue in different groups was compared using an unpaired student $t$-test. The data for DCF assay $\left(t_{1}=0\right.$ min vs. $\left.t_{2}=15 \mathrm{~min}\right)$ in each brain region was analyzed utilizing a 2-tailed paired $t$-test, while the comparison in the rate of ROS generation from different regions was performed by 2 -tailed unpaired $t$-test. In each case, the $t$-test was followed by a Bonferroni/Dunn test for multiple comparisons. All values are expressed as the mean values \pm the standard error of the mean of ' $n$ ' observations, and a probability level of $P<0.05$ was considered significant.

\section{RESULTS}

\subsection{NP-Induced Lesions in the Rat Striatum}

Four days after 3NP (20 mg/kg/day, i.p.) administration, all animals demonstrated behavioral abnormalities associated with 3NP toxicity, characterized by recumbence, dystonia and lethargy similar to those previouslydescribed [13,57-59]. The saline treated specimens did not display behavioral impairment throughout the duration of experiments. Upon examination with cresyl violet staining, 24 and 48 hours of 3NP treatment did not result in any apparent cell loss in any regions of the brain. However, three days following the first $3 \mathrm{NP}$ injection, two out of six rats had developed mild to moderate cell loss in the lateral striatum. Four days following 3NP treatment, one rat had moderate pathology, while the remaining rats displayed more severe neuronal loss, typically extending from lateral to dorsal and medial striatum (Figures 1(a) and (b)).

\subsection{3-Nitrotyrosine Levels}

In 3NP-treated rats, hematoxylin and eosin staining showed marked cellular alterations in the striatum characterized by nuclear shrinkage and the formation of perinuclear spaces (Figure 2(a)). Treatment of rats with 3NP for 48 hours did not result in the elevation of 3nitrotyrosine levels. However, three days following 3NP treatment, there was a mild elevation in 3-nitrotyrosine immunoreactivity in the striatum of some animals; nonetheless, upon quantitative Western blot analysis, the averaged results did not statistically differ from that of the saline control $(n=6, P=0.08)$. Following 4 days of $3 \mathrm{NP}$ treatment, the levels of 3-nitrotyrosine in the striatum were significantly greater than that of controls $(n=6$, $P<0.05)$. At this time point, there was a slight increase

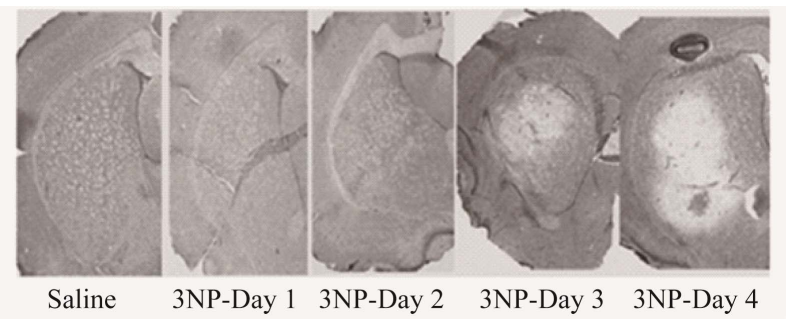

(a)

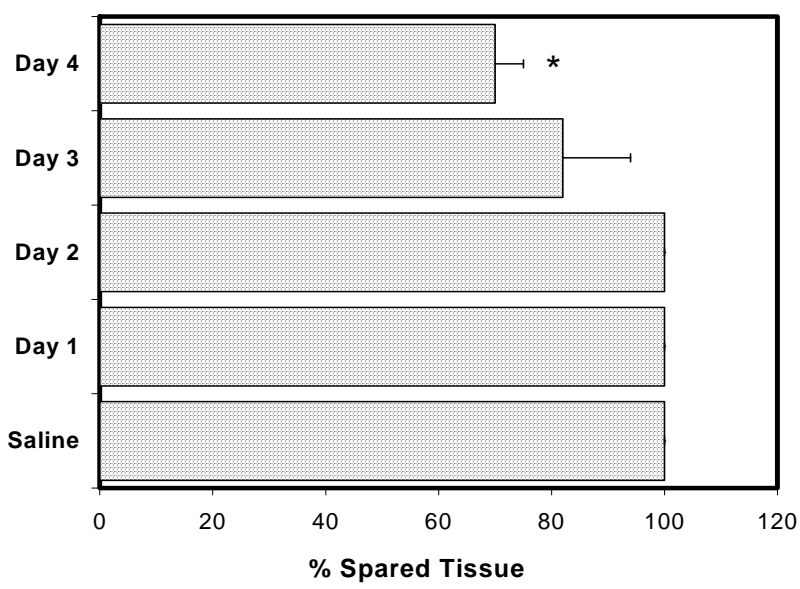

(b)

Figure 1. Intraperitoneal administration of $3 \mathrm{NP}$ results in selective striatal lesion. (a) Selective striatal neurodegeneration first appears in the lateral striatum and spreads throughout the dorsal and medial striatum; (b) Quantitative analysis of the percent spared tissue indicates a significant decrease in striatal spared tissue 4 days following $3 \mathrm{NP}$ treatment $(\mathrm{n}=6$ per day, $P<0.05)$.

in 3-nitrotyrosine immunoreactivity in the hippocampus, $(n=6, P=0.09)$ (Figure 2(c)). Cerebellum did not exhibit elevated 3-nitrotyrosine levels up to four days following 3NP treatment (Figures 2(b) and (c)).

\subsection{Poly ADP-Ribosylation Levels}

To investigate whether PARP-1 activation was associated with $3 \mathrm{NP}$-induced oxidative stress, we examined the levels of poly-ADP ribosylated proteins in various brain regions. There were occasional cells immunostained for poly-ADP ribosylated proteins in striatum and hippocampus following 4 days of 3NP treatment (data not shown). However, Western blot analysis did not indicate a significant increase in the total levels of poly ADP-ribosylated proteins (Figure 3).

\subsection{Poly (ADP-Ribose) Polymerase-1 Cleavage}

To further investigate the nature of PARP-1 involvement in the 3NP model, we utilized antibodies to detect PARP-1 cleavage products since it has previously been demonstrated that PARP-1 is cleaved during both apoptotic and necrotic cell death [46,60-62]. Twenty-four hours after $3 \mathrm{NP}$ administration, a weak $50 \mathrm{kDa}$ band was 

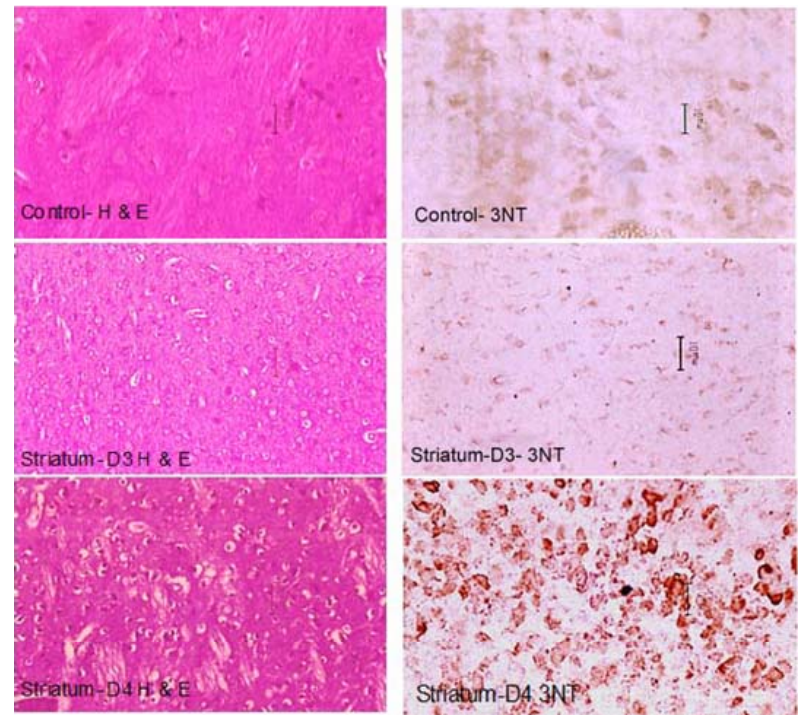

(a)

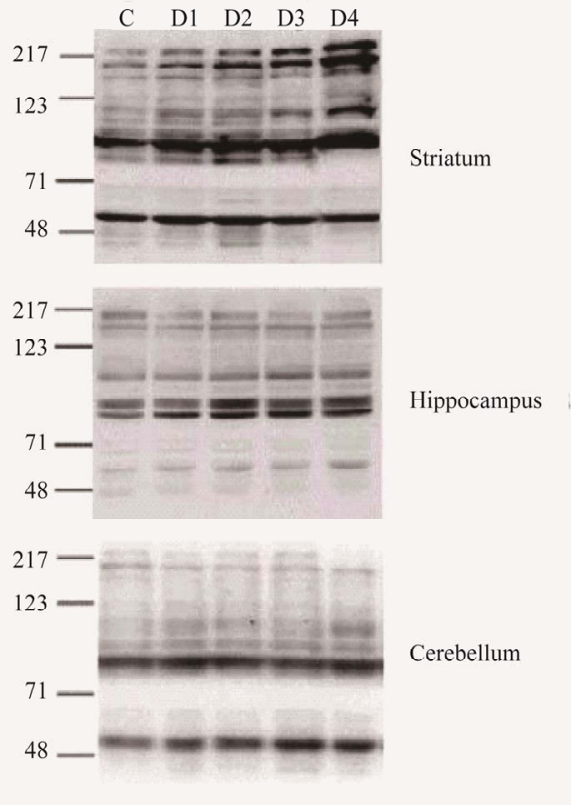

(b)

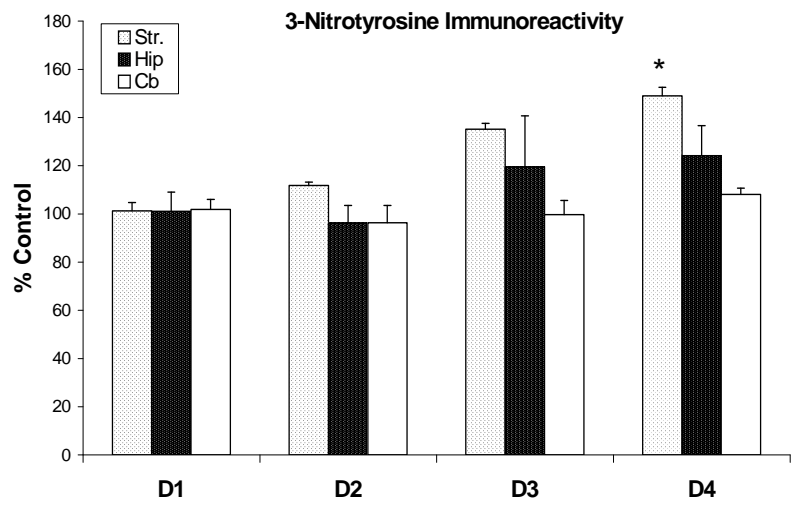

(c)

Figure 2. 3-Nitrotyrosine levels following 3NP treatment. (a) H \& E staining demonstrates histopathological characteristics of $3 \mathrm{NP}$ administration in the striatum marked by cellular alterations in the striatum and nuclear shrinkage and perinuclear spaces formation, while 3-nitrotyrosine immunoreactivity following 4 days of $3 \mathrm{NP}$ treatment indicates a robust increase in levels of 3-nitrotyrosine in striatum. No other regions examined exhibited a significant increase in 3-nitrotyrosine immunoreactivity as compared to the saline-treated controls; (b) Representative Western blot results for 3-nitrotyrosine in various brain regions; (c) Quantitative analysis of 3-nitrotyrosine levels in the corresponding region is illustrated as a percent of saline-treated controls $(n=6$ per day, $P<0.05) ;(\mathrm{C}=$ control, D = Day, 3NT = 3-nitrotyrosine, H \& E = hematoxylin and eosin stain, Scale bar $=10 \mu \mathrm{m}$, str = striatum, hip $=$ hippocampus, $\mathrm{cb}=$ cerebellum).

present in the striatum. The $50 \mathrm{kDa}$ fragment increased in intensity on the second and third day but was reduced by the end of the fourth day of treatment. Interestingly, in hippocampus, the $50 \mathrm{kDa}$ band did not appear until the third day and was present by the end of fourth day following 3NP administration. PARP-1 cleavage products were not observed in the cerebellum at any time points following 3NP (Figure 4).

\subsection{Mitochondrial ROS Production}

To investigate whether $3 \mathrm{NP}$ and calcium concentration in isolated mitochondria influences production of free radical species, we measured the generation of free radicals in isolated mitochondria from striatum and cerebellum of two- and ten-month old rats. In the older animals, 3NP did not increase DCF fluorescence in either striatum or cerebellum. In contrast, there was a reduction of DCF signal in the striatum and cerebellum of younger animals (Figure 5). The addition of two concentrations of $\mathrm{Ca}^{2+}$ $(50 \mu \mathrm{M}$ and $150 \mu \mathrm{M})$ to the isolated mitochondria preparation from the striatum of older animals resulted in a significant concentration-dependent increase in fluore- 


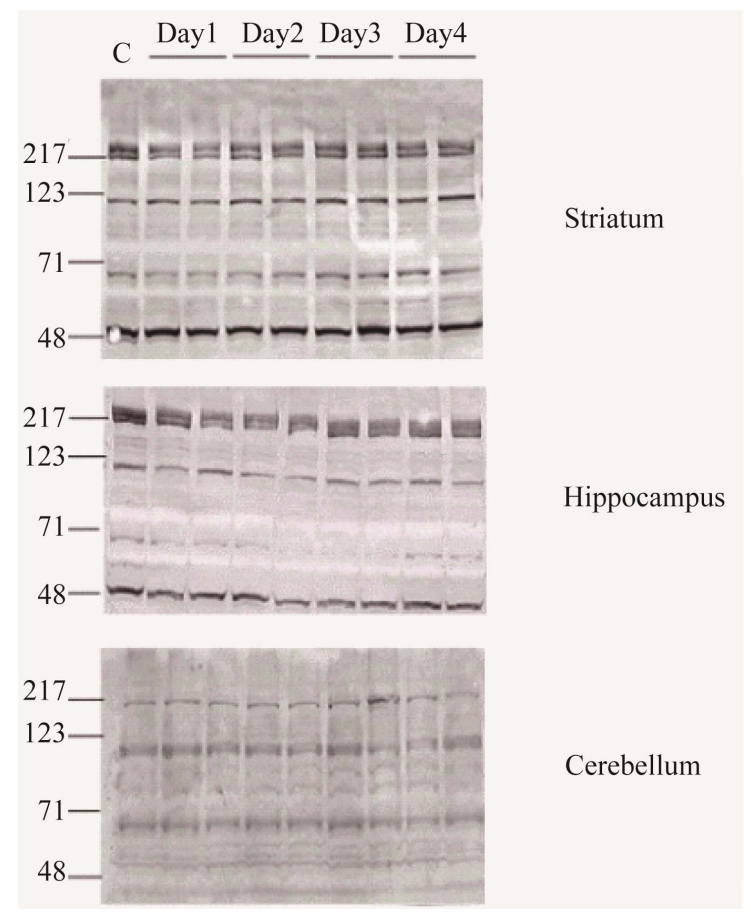

Figure 3. Analysis of poly ADP ribosylated proteins following 3NP treatment. Representative Western blots from various regions of brain demonstrate no major change in poly-ADP ribosylated proteins in any of the brain regions ( $n=6$ per day).

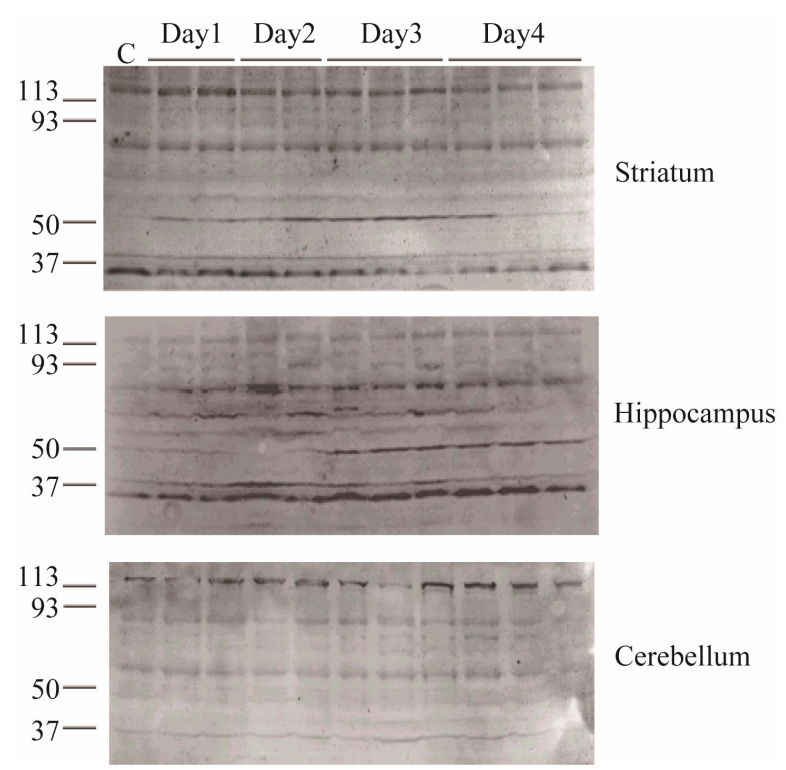

Figure 4. PARP-1 cleavage products following systemic administration of $3 \mathrm{NP}$. In the striatum, a $50 \mathrm{kDa}$ PARP-1 cleavage fragment first appears one day following $3 \mathrm{NP}$ treatment, intensifies on the second and third day and diminishes by the end of the fourth day, whereas in the hippocampus, a $50 \mathrm{kDa}$ PARP-1 fragment first appears on the third day post-3NP treatment and is sustained throughout the fourth day. In the cerebellum, there is no apparent increase in $50 \mathrm{kDa}$ fragment up to 4 days following $3 \mathrm{NP}$ treatment.

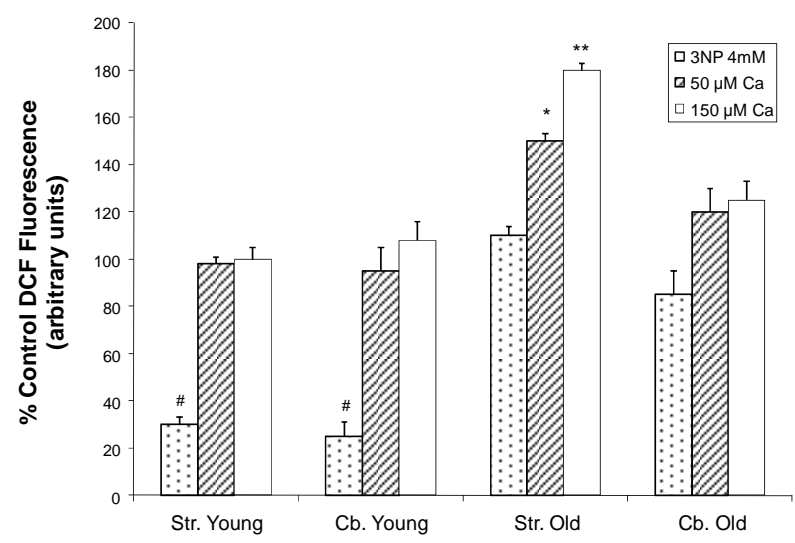

Figure 5. ROS production as detected by increased DCF fluorescence signal. $3 \mathrm{NP}$ administration $(4 \mathrm{mM})$ in mitochondrial preparation for 15 minutes did not result in a significant change in reactive oxygen species detected by DCF assay, while there was a significant decrease in DCF fluorescence signal in the striatum and cerebellum of the younger animals $\left({ }^{\sharp} P<0.01, n=6\right)$. The addition of calcium $(50 \mu \mathrm{M}$ and $150 \mu \mathrm{M})$ resulted in a significant calcium concentration-dependent increase in ROS production in the striatal mitochondrial preparation of the older animals $\left({ }^{*} P<0.05,{ }^{* *} P<0.01, n=6\right)$. However, similar concentrations of calcium did not alter DCF fluorescence signals in either the striatum or cerebellum of younger animals $(\mathrm{Ca}=$ Calcium, $\mathrm{Cb}=$ Cerebellum, $\mathrm{Str}=$ Striatum).

scence signal. Although there was an increase in ROS generation in cerebellar mitochondria in the aged animals, the increase was subtler when compared to the striatum (Figure 5). The striking finding was the lack of increase in DCF fluorescence in the presence of $\mathrm{Ca}^{2+}$ in the striatum of younger animals since mitochondria is the principal source of calcium-dependent free radical production $[47,48,63]$.

\section{DISCUSSION}

The acquired knowledge from natural cases of 3NP toxicity propelled a number of scientific studies to characterize this compound. The effort led to the discovery that chronic or acute administration of $3 \mathrm{NP}$ results in selective striatal degeneration among experimental animals $[19,20,25]$. Moreover, behavioral studies in primates indicated that $3 \mathrm{NP}$ produces cognitive and motor deficits reminiscent of those seen in Huntington's disease $[7,64,65]$. Several hypotheses have been proposed as the possible mechanisms for 3NP toxicity. In 1987, Hamilton and Gould observed the similarities between the neuronal damage in $3 \mathrm{NP}$ and that of kainic acid in the rat model. They proposed an excitotoxic mechanism [25]. Excitotoxicity has been associated with a number of pathological conditions, such as ischemia and hypoglycemia, as well as several chronic neurodegenerative diseases [66-69]. Furthermore, it has been demonstrated that excitotoxicity due to overexposure to excitatory amino 
acids can produce lesions in the CNS similar to those observed in Huntington's disease [70-72]. L-glutamate is the primary excitatory neurotransmitter in the central nervous system [73]. High doses of intrastriatal administration of glutamate result in rapid development of neuropathological features that are characterized by swelling of neuronal cell bodies and organelles, as well as nuclear pyknosis $[66,74]$. In addition, cell culture studies have signified the role of glutamate receptors in $3 \mathrm{NP}$ neurotoxicity following the reduction in intracellular energy levels and inhibition of ATP-dependent ion pumps $[24,29,75]$. These findings conform with the "secondary excitotoxicity" hypothesis stating that energy impairment indirectly results in the opening of glutamate receptors, influx of massive amount of calcium and overwhelming the mitochondrial calcium buffering capacity, ultimately leading to the activation of cell death pathways [24,29, 70,75-78]. Moreover, Liot and colleagues have established temporal relationship of 3NP administration and ROS production [79]. They documented an instant drop in ATP, and several hours after the initial injection, a significant increase in ROS production. A possible explanation for this phenomenon was that the ATP drop and ROS production, lead to depolarization of the plasma membrane, possibly relieving the $\mathrm{Mg}^{2+}$ block of voltagegated NMDA receptors. This event leads to the opening of the NMDA receptors, allowing massive $\mathrm{Ca}^{2+}$ influx, ultimately resulting in substantial ROS production.

Other reports have implicated oxidative stress as a possible pathway in 3NP neurotoxicity since following systemic $3 \mathrm{NP}$ administration, there is an increase in protein oxidation markers in the striatum $[80,81]$. The exposure of living organisms to free radicals and oxidants is part of an intricate nature of aerobic respiration. Every cell in the human body has mechanisms in place to counteract or even utilize the production of free radicals to conduct a variety of cellular functions. Thus, a correct definition of oxidative stress as a pathological event is considered necessary. An excellent definition for oxidative stress was provided by Sies and Cadenas as "the critical disturbances in prooxidant-antioxidant balance in favor of the former" [82]. Consequently, the occurrence of oxidative challenge in cells does not necessarily constitute oxidative stress. However, when the noxious external or internal sources enhance the production of oxidants, and the cellular defensive mechanisms against the oxidants are either overwhelmed or compromised, we approach a pathological oxidative environment which leads to oxidative stress and subsequently oxidative damage [83].

3-Nitrotyrosine formation has previously been reported as a reliable marker for protein oxidation in the $3 \mathrm{NP}$ model $[80,84-86]$ and other neurodegenerative diseases [87-89]. 3-Nitrotyrosine is the product of reaction between nitrogen species with tyrosine residues of proteins [90]. The nitration of tyrosine residues alters the conformation of proteins, resulting in a change of function such as the ability to be phosphorylated [91]. Since the nitration of tyrosine residues is an irreversible process, abnormal increases in 3-nitrotyrosine levels acutely interfere with routine cellular functions [92]. One likely source of elevated 3-nitrotyrosine is peroxynitrite (ONOO-), a strong oxidant capable of oxidizing proteins and DNA. In an oxidative environment, peroxynitrite can be readily formed by the interaction between nitric oxide and superoxide radicals [93]. Interestingly, the inhibition of mitochondrial aerobic respiration, as seen in the $3 \mathrm{NP}$ model, results in the loss of mitochondrial electro-chemical gradient and subsequently excessive generation of superoxide radicals [94]. The increase in superoxide radicals accompanied with the excess production of nitric oxide may account for peroxynitrite and subsequent 3nitrotyrosine elevation. Our results demonstrate that the levels of protein oxidation marker, 3-nitrotyrosine, significantly increase in the striatum, the primary site of neurodegeneration in 3NP model. However, in absence of increased 3-nitrotyrosine prior to the formation of striatal lesion, it is difficult to conclude that the increased levels of 3-nitrotyrosine, and in turn, protein oxidation are causative agents in 3NP-induced striatal degeneration. The current results suggest that oxidative stress, as detected by elevated levels of 3-nitrotyrosine, coincides but does not necessarily precedes the selective striatal degeneration. In addition, the delayed elevation of 3-nitrotyrosine in hippocampus is also consistent with the reports demonstrating a delayed hippocampal lesion following 3NP treatment [95].

Although immunohistochemical analysis indicated few cells stained strongly for poly ADP-ribosylated proteins, quantitative analysis of poly ADP-ribosylated proteins in the regions of interest (striatum, hippocampus and cerebellum) revealed no significant quantitative increase in poly ADP-ribosylated proteins. Proteolytic cleavage of PARP-1 has been widely utilized as specific biochemical marker for DNA oxidation. Kaufmann and colleagues first reported that PARP-1 is a substrate for caspases 3 and 7 and suggested that the proteolytic cleavage of PARP-1 can be utilized as a biochemical hallmark of apoptosis [96]. PARP-1 cleavage by caspases produces two major 89 and $24 \mathrm{kDa}$ fragments as a result of cleavage in N-terminal DNA binding domain [96,97]. Interestingly, Shah and colleagues demonstrated that PARP-1 is also cleaved during the necrotic death, producing a major $50 \mathrm{kDa}$ fragment [62]. Subsequent studies by Gobeil and colleagues demonstrated that PARP-1 is also a substrate for cathepsins B and G, two major lysosomal proteases $[60,62]$. Since in the necrotic cell death the content of lysosomes are released into the cytosol 
[98], it is likely that the lack of a robust increase in poly ADP-ribosylation in the striatum is due to the cleavage of PARP-1 by lysosomal proteases and subsequent inability of PARP-1 to polymerize the ADP-ribose polymers on target proteins. Furthermore, PARP-1 is a highly energy-dependent enzyme, which suggests that in metabolic impairment paradigms, such as the 3NP model in which both the Krebs cycle and the electron transport chain are compromised, PARP-1 function may be inhibited systemically due to inadequate availability of energy resources. In our experimental paradigm, one day post 3NP administration, Western blot analysis of PARP-1 cleavage products demonstrated a $50 \mathrm{kDa}$ fragment in the striatum, while the similar PARP-1 cleavage fragment did not appear in the hippocampus until the third day following 3NP. No major PARP-1 cleavage products were detected in the cerebellum. These results are interesting in the light of previous reports indicating that the striatum is the first site of neurodegeneration followed by hippocampal degeneration after longer treatment with 3NP [19,22,99,100].

Although Calcium has no direct effect on oxidative phosphorylation (for review see [52]), imbalance in calcium homeostasis have been implicated in 3NP-induced toxicity [53]. Calcium, a divalent cation, is the fifth most common element in the mammalian body [101]. Calcium can be used by cells as a second messenger to control a plethora of cellular functions including proliferation, contraction, secretion, neuronal excitation and cell death among many others. In its insoluble form, calcium exists as crystalline hydroxyapatite in bones and teeth, whereas in its soluble form calcium is the prime inorganic second messenger for regulation of the cellular functions. The cytosolic level of $\mathrm{Ca}^{2+}$ is kept low $(10-100 \mathrm{nM})$ but normal physiological stimulations result in the level increasing up to $500-1000 \mathrm{nM}$ [102]. In neurons, calcium determines the activity of adenylate cyclase and phosphodiesterase through reversible interaction with calmodulin [103]. Calcium also serves to regulate membrane permeability, allowing neurotransmitter release as well as diminishing neuromuscular excitability. Within cells, only a small fraction of total cellular calcium (approximately $0.1 \%$ ) is found free in the cytosol, the majority of calcium is primarily bound to proteins and nucleic acids or is sequestered in endoplasmic reticulum and mitochondria [102]. Mitochondria have a high capacity for transient calcium storage. In an excellent example of evolutionary adaptation, unlike endoplasmic reticulum, the mitochondrial $\mathrm{Ca}^{2+}$ levels under normal physiological condition are similar to that of the cytosol $[104,105]$. The main route of calcium entry into mitochondria is via the $\mathrm{Ca}^{2+}$ uniporter. The $\mathrm{Ca}^{2+}$ influx into mitochondria via $\mathrm{Ca}^{2+}$ uniporter is driven by the mitochondrial membrane potential which creates a negatively charged mito-chon- drial matrix, attracting positively charged $\mathrm{Ca}^{2+}$ ions. Interestingly, $\mathrm{Ca}^{2+}$ efflux from mitochondria is regulated via $\mathrm{Na}^{+} / \mathrm{Ca}^{2+}$ pumps by which $\mathrm{Na}^{+}$entry into mitochondria is coupled with $\mathrm{Ca}^{2+}$ efflux from mitochondria. The $\mathrm{Ca}^{2+}$ influx into mitochondria is gradient-dependent; the higher cytosolic calcium levels, the larger the rate of calcium entry into the mitochondria. In contrast, the rate of efflux of calcium via $\mathrm{Na}^{+} / \mathrm{Ca}^{2+}$ pumps is constant. In other words, if the cytosolic $\mathrm{Ca}^{2+}$ levels increase, more $\mathrm{Ca}^{2+}$ enters mitochondria, while the rate of efflux remains the same, and as a result, cytosolic calcium levels decrease, avoiding cytotoxic effects associated with large cytosolic calcium concentration. When cytosolic $\mathrm{Ca}^{2+}$ levels decrease, the influx of $\mathrm{Ca}^{2+}$ via the uniporter also decreases, while the rate of efflux remains constant, allowing the cytosolic $\mathrm{Ca}^{2+}$ levels to increase and reach the physiological concentration [106]. Considering the intricate mechanisms in place to regulate $\mathrm{Ca}^{2+}$ concentration in cells, it is expected that any major disturbances in this system result in tissue injury and cell death. Numerous reports have demonstrated that the disruption in cellular $\mathrm{Ca}^{2+}$ homeostasis, either due to excitotoxicity or oxidative stress, ultimately results in cellular demise [107111]. However, the isolated mitochondrial analysis as reported in the current study suggest that aging results in striking differences in mitochondrial free radical production in response to calcium. This conclusion was reached by demonstrating that calcium elevation in isolated mitochondria from the older animals caused a significant increase ROS generation as compared to that of the younger animals. Although $3 \mathrm{NP}$ alone does not directly stimulate free radical production, the metabolic impairment induced by 3NP interferes with the intracellular $\mathrm{Ca}^{2+}$ homeostasis. This finding is of significant importance since it suggests that in aging, mitochondria become more susceptible to the generation of reactive oxygen species, and in conditions that cause a concurrent imbalance in mitochondrial calcium homeostasis, the effects may be magnified.

The current study implicates mitochondria dysfunction as a key cellular target in pathological states that are associated with metabolic impairment. Moreover, the results also reinforce the notion that mitochondrial function in the striatum and cerebellum respond differently to the aging process, which may explain the variable regional vulnerability in $3 \mathrm{NP}$ model. Taken together, the variable response of the isolated mitochondria from striatum and cerebellum of aged animals to 3NP toxicity and calcium overload suggest more acute changes in the older specimens. This finding suggests an intrinsic difference in the function of mitochondria in respect to age and anatomical location. Consistent with this hypothesis, previous reports have indicated a decline in mitochondrial function with age $[112,113]$. Others have shown oxidized 
hydroethidine, a molecule used to measure intracellular superoxide anion presence, increases with age [114]. Moreover, age-related decline in levels of glutathione, a molecule involved in the function of antioxidant enzymes, has been documented [115]. The striking difference in mitochondria ROS generation in response to calcium addition suggests that mitochondrial calcium homeostasis in the older rats may play a significant role in ROS production. This finding suggests the neurodegenerative processes in 3NP model may involve alternative pathways in younger and older animals and offers further explanation for likely mechanisms involved in the age-dependent variability seen in 3NP model.

Previous studies have shown that $3 \mathrm{NP}$ causes more damage to the striatum than to other regions of the brain $[116,117]$. However, $3 \mathrm{NP}$ causes similar levels of SDH inhibition in different regions of the brain [22-23]. Striatal sensitivity cannot be only attributed to SDH inhibition; therefore, other factors must be considered. One possible mechanism that may account for the preferential striatal vulnerability is suggested to be a selective breakdown of the striatal blood brain barrier, thereby allowing a higher concentration of $3 \mathrm{NP}$ to reach the striatum [118119]. Other reports have implicated $\mathrm{Ca}^{2+}$ in the preferential striatal neurodegeneration. The typical mitochondrial response to $\mathrm{Ca}^{2+}$ administration is sustained membrane depolarization, immediate increase in respiration, and mitochondrial swelling [120]. Striatal mitochondria seem to be more sensitive to intracellular $\mathrm{Ca}^{2+}$ levels than their cortical counterparts [121]. A possible explanation for the sensitivity of striatal mitochondria to intracellular $\mathrm{Ca}^{2+}$ could be explained by a component of mitochondria membrane transition pore, cyclophilin D (CyP-D), which its concentration is almost two fold greater in striatum than in cortical mitochondria [121]. The link between CyP-D and $\mathrm{Ca}^{2+}$ was demonstrated in a study of liver mitochondria from mice, in which the $\mathrm{Ca}^{2+}$ sensitivity of mice with CyP-D was compared to that of mutant mice without CyP-D. The study also showed that the mutant mice needed twice the amount of $\mathrm{Ca}^{2+}$, compared to the wild-type, to open mitochondrial membrane transition pore [122]. Another characteristic of the striatum is that it is heavily innervated with glutamate inputs from the cerebral cortex. However, glutamate alone cannot account for striatal sensitivity, because one would expect other brain regions with heavy glutamatergic inputs to be highly susceptible to 3NP (i.e. Purkinje cells in the cerebellum). Even though lesions have been observed in the hippocampus, studies show extrastriatal regions do not seem as sensitive to $3 \mathrm{NP}$ [21,123-126]. The striatum also receive dopamine inputs from the substantianigra. Reynolds and colleagues have demonstrated that removal of dopamine input from one striatum prevented 3NP lesions in the denervated striatum but not in the contralateral striatum [126]. Furthermore, dopamine and 3NP have been shown to increase the generation of hydroxyl radi$\mathrm{cal}$; however, when administered together, they work synergistically [127]. In addition, the endogenous dopamine, in the presence of $3 \mathrm{NP}$, decreases striatal mitochondria oxygen consumption and increases superoxide levels in synaptosomes [128].

In summary, from studies to date, there is considerable evidence suggesting that energy impairment is a common biochemical mechanism underlying the etiology of a number of neurodegenerative disorders [129-130]. In the current study, we utilized 3NP to model energy impairment in rodents. The elevated level of 3-nitrotyrosine in the striatum coincides with the development of striatal lesion. However, it is unclear whether oxidative damage is an effector or a consequence of the neurodegeneration. The lack of robust increased levels of ADP-ribosylated proteins is likely due to the cleavage and subsequent deactivation of PARP-1 and does not exclude the role of oxidative stress in 3NP toxicity. Although $3 \mathrm{NP}$ does not directly increase the production of free radicals, the addition of calcium results in a significant increase in the production of free radical species in the mitochondria of older animals. The results also suggest that in aging, mitochondria become more susceptible to the generation of reactive oxygen species, which also may become greater in conditions that cause a concurrent imbalance in mitochondrial calcium homeostasis.

\section{ACKNOWLEDGEMENTS}

"Authors thank and acknowledge Professor James W. Geddes for his assistance, expertise and invaluable advices".

\section{REFERENCE}

[1] James, L.F., et al. (1980) Field and experimental studies in cattle and sheep poisoned by nitro-bearing Astragalus or their toxins. American Journal of Veterinary Research, 41, 377-382.

[2] Hu, W.J. (1986) Isolation and structure determination of arthrinium toxin causing sugarcane poisoning. Nitropropionic Acid, 20, 321-323.

[3] Alston, T.A., Mela, L. and Bright, H.J. (1977) 3-nitropropionate, the toxic substance of Indigofera, is a suicide inactivator of succinate dehydrogenase. Proceedings of the National Academy of Sciences of the United States, 74, 3767-3771. doi:10.1073/pnas.74.9.3767

[4] Ludolph, A.C., et al. (1991) 3-nitropropionic acid-exogenous animal neurotoxin and possible human striatal toxin. Canadian Journal of Neurological Sciences, 18, 492-498.

[5] Borlongan, C.V., et al. (1997) Hyperactivity and hypoactivity in a rat model of Huntington's disease: The systemic 3-nitropropionic acid model. Brain Research Protocols, 1, 253-257. doi:10.1016/S1385-299X(96)00037-2 
[6] Borlongan, C.V., Koutouzis, T.K. and Sanberg, P.R. (1997) 3-Nitropropionic acid animal model and Huntington's disease. Neuroscience \& Biobehavioral Reviews, 21, 289293. doi:10.1016/S0149-7634(96)00027-9

[7] Beal, M.F. (1994) Neurochemistry and toxin models in Huntington's disease. Current Opinion in Neurology, 7, 542-547. doi:10.1097/00019052-199412000-00012

[8] Palfi, S., et al. (1996) Chronic 3-nitropropionic acid treatment in baboons replicates the cognitive and motor deficits of Huntington's disease. The Journal of Neuroscience, 16, 3019-3025.

[9] He, F., et al. (1990) Mycotoxin-induced encephalopathy and dystonia in children. Taylor and Francis, London.

[10] He, F., et al. (1995) Delayed dystonia with striatal CT lucencies induced by a mycotoxin (3-nitropropionic acid). Neurology, 45, 2178-2183. doi:10.1212/WNL.45.12.2178

[11] Ming, L. (1995) Moldy sugarcane poisoning-A case report with a brief review. Journal of ToxicologyClinical Toxicology, 33, 363-367. doi:10.3109/15563659509028924

[12] Borlongan, C.V., et al. (1995) Systemic 3-nitropropionic acid: Behavioral deficits and striatal damage in adult rats. Brain Research Bulletin, 36, 549-556. doi:10.1016/0361-9230(94)00242-S

[13] Borlongan, C.V., et al. (1995) Behavioral pathology induced by repeated systemic injections of 3-nitropropionic acid mimics the motoric symptoms of Huntington's. Brain Research, 697, 254-257. doi:10.1016/0006-8993(95)00901-2

[14] Nasr, P., Carbery, T. and Geddes, J.W. (2009) N-methylD-aspartate receptor antagonists have variable affect in 3-nitropropionic acid toxicity. Neurochemical Research, 34, 490-498. doi:10.1007/s11064-008-9809-3

[15] Butler, A.K., Uryu, K. and Chesselet, M.F. (1998) A role for N-methyl-D-aspartate receptors in the regulation of synaptogenesis and expression of the polysialylated form of the neural cell adhesion molecule in the developing striatum. Developmental Neuroscience, 20, 253-262. doi:10.1159/000017319

[16] Parent, A. and Hazrati, L.N. (1995) Functional anatomy of the basal ganglia. I. The cortico-basal ganglia-thalamo-cortical loop. Brain Research Reviews, 20, 91-127. doi:10.1016/0165-0173(94)00007-C

[17] Parent, A. and Hazrati, L.N. (1995) Functional anatomy of the basal ganglia. II. The place of subthalamic nucleus and external pallidum in basal ganglia circuitry. Brain Research Reviews, 20, 128-154. doi:10.1016/0165-0173(94)00008-D

[18] Yung, K.K., et al. (1995) Immunocytochemical localization of D1 and D2 dopamine receptors in the basal ganglia of the rat: Light and electron microscopy. Neuroscience, 65, 709-730. doi:10.1016/0306-4522(94)00536-E

[19] Beal, M.F., et al. (1993) Neurochemical and histologic characterization of striatal excitotoxic lesions produced by the mitochondrial toxin 3-nitropropionic acid. The Journal of Neuroscience, 13, 4181-4192.

[20] Brouillet, E., et al. (1993) Age-dependent vulnerability of the striatum to the mitochondrial toxin 3-nitropropionic acid. Journal of Neurochemistry, 60, 356-359. doi:10.1111/j.1471-4159.1993.tb05859.x

[21] Bossi, S.R., Simpson, J.R. and Isacson, O. (1993) Age dependence of striatal neuronal death caused by mitochondrial dysfunction. Neuroreport, 4, 73-76. doi:10.1097/00001756-199301000-00019

[22] Pang, Z., Umberger, G.H. and Geddes, J.W. (1996) Neuronal loss and cytoskeletal disruption following intrahippocampal administration of the metabolic inhibitor malonate: Lack of protection by MK-801. Journal of Neurochemistry, 66, 474-484.

doi:10.1046/j.1471-4159.1996.66020474.x

[23] Brouillet, E., et al. (1998) Partial inhibition of brain succinate dehydrogenase by 3-nitropropionic acid is sufficient to initiate striatal degeneration in rat. Journal of Neurochemistry, 70, 794-805. doi:10.1046/j.1471-4159.1998.70020794.x

[24] Novelli, A., et al. (1988) Glutamate becomes neurotoxic via the N-methyl-D-aspartate receptor when intracellular energy levels are reduced. Brain Research, 451, 205-212. doi:10.1016/0006-8993(88)90765-2

[25] Hamilton, B.F. and Gould, D.H. (1987) Nature and distribution of brain lesions in rats intoxicated with 3-nitropropionic acid: A type of hypoxic (energy deficient) brain damage. Acta Neuropathologica (Berlin), 72, 286-297. doi:10.1007/BF00691103

[26] Binienda, Z., et al. (1998) Effect of acute exposure to $3-$ nitropropionic acid on activities of endogenous anti-oxidants in the rat brain. Neuroscience Letters, 251, 173176. doi:10.1016/S0304-3940(98)00539-4

[27] Kim, G.W., et al. (2000) Excitotoxicity is required for induction of oxidative stress and apoptosis in mouse striatum by the mitochondrial toxin, 3-nitropropionic acid. Journal of Cerebral Blood Flow \& Metabolism, 20, 119. 129. doi:10.1097/00004647-200001000-00016

[28] Zeevalk, G.D., L.P. Bernard, and W.J. Nicklas, Oxidative stress during energy impairment in mesencephalic cultures is not a downstream consequence of a secondary excitotoxicity. Neuroscience, 96, 309-316. doi:10.1016/S0306-4522(99)00567-9

[29] Pang, Z. and Geddes, J.W. (1997) Mechanisms of cell death induced by the mitochondrial toxin 3-nitropropionic acid: Acute excitotoxic necrosis and delayed apoptosis. The Journal of Neuroscience, 17, 3064-3073.

[30] Brenman, J.E. and Bredt, D.S. (1997) Synaptic signaling by nitric oxide. Current Opinion in Neurobiology, 7, 374378. doi:10.1016/S0959-4388(97)80065-7

[31] Christopherson, K.S., et al. (1999) PSD-95 assembles a ternary complex with the N-methyl-D-aspartic acid receptor and a bivalent neuronal NO synthase PDZ domain. The Journal of Biological Chemistry, 274, 27467-27473. doi:10.1074/jbc.274.39.27467

[32] Sattler, R., et al. (1999) Specific coupling of NMDA receptor activation to nitric oxide neurotoxicity by PSD-95 protein. Science, 284, 1845-1848. doi:10.1126/science.284.5421.1845

[33] Moncada, S. and Palmer, R.M. (1991) Biosynthesis and actions of nitric oxide. Seminars in Perinatology, 15, 16- 
19.

[34] Knowles, R.G. and Moncada, S. (1994) Nitric oxide synthases in mammals. Biochemical Journal, 298, 249-258.

[35] Darley-Usmar, V., Wiseman, H. and Halliwell, B. (1995) Nitric oxide and oxygen radicals: A question of balance. FEBS Letters, 369, 131-135. doi:10.1016/0014-5793(95)00764-Z

[36] Zweier, J.L., et al. (1995) Enzyme-independent formation of nitric oxide in biological tissues. Nature Medicine, $\mathbf{1}$, 804-809. doi:10.1038/nm0895-804

[37] Farinati, F., et al. (1996) Gastric antioxidant, nitrites, and mucosal lipoperoxidation in chronic gastritis and Helicobacter pylori infection. Journal of Clinical Gastroenterology, 22, 275-281.

doi:10.1097/00004836-199606000-00007

[38] Tamir, S. and Tannenbaum, S.R. (1996) The role of nitric oxide (NO.) in the carcinogenic process. Biochimica et Biophysica Acta, 1288, F31-F36.

[39] Beckman, J.S. and Koppenol, W.H. (1996) Nitric oxide, superoxide, and peroxynitrite: The good, the bad, and ugly. American Journal of Physiology, 271, C1424-C1437.

[40] Pannala, A.S., et al. (1998) Inhibition of peroxynitrite dependent tyrosine nitration by hydroxycinnamates: Nitration or electron donation. Free Radical Biology \& Medicine, 24, 594-606. doi:10.1016/S0891-5849(97)00321-3

[41] Beckman, J.S. (1996) Oxidative damage and tyrosine nitration from peroxynitrite. Chemical Research in Toxicology, 9, 836-844. doi:10.1021/tx9501445

[42] Schulz, J.B., Matthews, R.T. and Beal, M.F. (1995) Role of nitric oxide in neurodegenerative diseases. Current Opinion in Neurology, 8, 480-486. doi:10.1097/00019052-199512000-00016

[43] Ischiropoulos, H. (1998) Biological tyrosine nitration: A pathophysiological function of nitric oxide and reactive oxygen species. Archives of Biochemistry and Biophysics, 356, 1-11. doi:10.1006/abbi.1998.0755

[44] Calabrese, V., Bates, T.E. and Stella, A.M. (2000) NOsynthase and NO-dependent signal pathways in brain aging and neurodegenerative disorders: The role of oxidant/antioxidant balance. Neurochemical Research, 25, 1315-1341. doi:10.1023/A:1007604414773

[45] Calabrese, V., et al. (2002) Nitric oxide synthase is present in the cerebrospinal fluid of patients with active multiple sclerosis and is associated with increases in cerebrospinal fluid protein nitrotyrosine and S-nitrosothiols and with changes in glutathione levels. Journal of Neuroscience Research, 70, 580-587. doi:10.1002/jnr.10408

[46] Ha, H.C. and Snyder, S.H. (2000) Poly (ADP-ribose) polymerase-1 in the nervous system. Neurobiology of Disease, 7, 225-39. doi:10.1006/nbdi.2000.0324

[47] Reynolds, I.J. and Hastings, T.G. (1995) Glutamate induces the production of reactive oxygen species in cultured forebrain neurons following NMDA receptor activation. The Journal of Neuroscience, 15, 3318-3327.

[48] Dugan, L.L., et al. (1995) Mitochondrial production of reactive oxygen species in cortical neurons following exposure to N-methyl-D-aspartate. The Journal of Neuro- science, 15, 6377-6388.

[49] Cadenas, E. and Boveris, A. (1980) Enhancement of hydrogen peroxide formation by protophores and ionophores in antimycin-supplemented mitochondria. Biochemical Journal, 188, 31-37.

[50] Sousa, S.C., et al. (2003) $\mathrm{Ca}^{2+}$-induced oxidative stress in brain mitochondria treated with the respiratory chain inhibitor rotenone. FEBS Letters, 543, 179-183. doi:10.1016/S0014-5793(03)00421-6

[51] Votyakova, T.V. and Reynolds, I.J. (2005) $\mathrm{Ca}^{2+}$-induced permeabilization promotes free radical release from rat brain mitochondria with partially inhibited complex I. The Journal of Neuroscience, 93, 526-537. doi:10.1111/j.1471-4159.2005.03042.x

[52] Peng, T.I. and Jou, M.J. (2010) Oxidative stress caused by mitochondrial calcium overload. Annals of the New York Academy of Sciences, 1201, 183-188. doi:10.1111/j.1749-6632.2010.05634.x

[53] Jacquard, C., et al. (2006) Brain mitochondrial defects amplify intracellular $\left[\mathrm{Ca}^{2+}\right]$ rise and neurodegeneration but not $\mathrm{Ca}^{2+}$ entry during NMDA receptor activation. The FASEB Journal, 20, 1021-1023. doi:10.1096/fj.05-5085fje

[54] Laemmli, U.K. (1970) Cleavage of structural proteins during the assembly of the head of bacteriophage T4. Nature, 227, 680-685. doi:10.1038/227680a0

[55] Sullivan, P.G., Thompson, M.B., and Scheff, S.W. (1999) CyclosporinA attenuates acute mitochondrial dysfunction following traumatic brain injury. Experimental Neurology, 160, 226-234. doi:10.1006/exnr.1999.7197

[56] Sullivan, P.G., Geiger, J.D., Mattson, M.P., and Scheff, S.W. (2000) Dietary supplement creatine protects against traumatic brain injury. Annals of Neurology, 48, 723-729. doi:10.1002/1531-8249(200011)48:5<723::AID-ANA5> 3.0.CO;2-W

[57] Koutouzis, T.K., et al. (1994) Systemic 3-nitropropionic acid: Long-term effects on locomotor behavior. Brain Research, 646, 242-246.

doi:10.1016/0006-8993(94)90085-X

[58] Koutouzis, T.K., et al. (1994) Intrastriatal 3-nitro-propionic acid: A behavioral assessment. Neuroreport, 5, 22412245. doi:10.1097/00001756-199411000-00009

[59] Borlongan, C.V., et al. (1995) Systemic 3-nitropropionic acid: Behavioral deficits and striatal damage in adult rats. Brain Research Bulletin, 36, 549-556. doi:10.1016/0361-9230(94)00242-S

[60] Gobeil, S., et al. (2001) Characterization of the necrotic cleavage of poly(ADP-ribose) polymerase (PARP-1): Implication of lysosomal proteases. Cell Death Differ, 8, 588594. doi:10.1038/sj.cdd.4400851

[61] Ha, H.C. and Snyder, S.H. (1999) Poly (ADP-ribose) polymerase is a mediator of necrotic cell death by ATP depletion. Proceedings of the National Academy of Sciences, 96, 13978-13982. doi:10.1073/pnas.96.24.13978

[62] Shah, G.M., Shah, R.G. and Poirier, G.G. (1996) Different cleavage pattern for poly (ADP-ribose) polymerase during necrosis and apoptosis in HL-60 cells. Biochemi- 
cal and Biophysical Research Communications, 229, 838844. doi:10.1006/bbrc.1996.1889

[63] Perez Velazquez, J.L., Frantseva, M.V. and Carlen, P.L. (1997) In vitro ischemia promotes glutamate-mediated free radical generation and intracellular calcium accumulation in hippocampal pyramidal neurons. The Journal of Neuroscience, 17, 9085-9094.

[64] Tunez, I., et al. (2010) 3-Nitropropionic acid as a tool to study the mechanisms involved in Huntington's disease: Past, present and future. Molecules, 15, 878-916. doi: $10.3390 /$ molecules 15020878

[65] Wu, C.L., et al. (2010) Neuroprotective mechanisms of brain-derived neurotrophic factor against 3-nitropropionic acid toxicity: Therapeutic implications for Huntington's disease. Annals of the New York Academy of Sciences, 1201, 8-12. doi:10.1111/j.1749-6632.2010.05628.x

[66] Choi, D.W. (1992) Excitotoxic cell death. Journal of Neurobiology, 23, 1261-1276. doi:10.1002/neu.480230915

[67] Coyle, J.T., et al. (1981) Excitatory amino acid neurotoxins: Selectivity, specificity, and mechanisms of action. Based on an NRP one-day conference held June 30, 1980. Neurosciences Research Program Bulletin, 19, 1-427.

[68] Rhee, S.G., et al. (1991) Multiple forms of phosphoinositide-specific phospholipase $\mathrm{C}$ and different modes of activation. Biochemical Society Transactions, 19, 337-341.

[69] Rothman, S.M. and Olney, J.W. (1986) Glutamate and the pathophysiology of hypoxic-ischemic brain damage. Annals of Neurology, 19, 105-111. doi:10.1002/ana.410190202

[70] Beal, M.F. (1992) Mechanisms of excitotoxicity in neurologic diseases. FASEB Journal, 6, 3338-3344.

[71] Coyle, J.T. and Puttfarcken, P. (1993) Oxidative stress, glutamate, and neurodegenerative disorders. Science, 262, 689-695. doi:10.1126/science.7901908

[72] DiFiglia, M. (1990) Excitotoxic injury of the neostriatum: A model for Huntington's disease. Trends in Neurosciences, 13, 286-289. doi:10.1016/0166-2236(90)90111-M

[73] Westerberg, E., et al. (1987) Excitatory amino acid receptors and ischemic brain damage in the rat. Neuroscience Letters, 73, 119-124. doi:10.1016/0304-3940(87)90004-8

[74] Olney, J.W. (1971) Glutamate-induced neuronal necrosis in the infant mouse hypothalamus. An electron microscopic study. Journal of Neuropathology \& Experimental Neurology, 30, 75-90. doi:10.1097/00005072-197101000-00008

[75] Zeevalk, G.D. and Nicklas, W.J. (1991) Mechanisms underlying initiation of excitotoxicity associated with metabolic inhibition. Journal of Pharmacology and Experimental Therapeutics, 257, 870-878.

[76] Albin, R.L. and Greenamyre, J.T. (1992) Alternative excitotoxic hypotheses. Neurology, 42, 733-738. doi:10.1212/WNL.42.4.733

[77] Beal, M.F. (1992) Does impairment of energy metabolism result in excitotoxic neuronal death in neurodegenerative illnesses. Annals of Neurology, 31, 119-130. doi:10.1002/ana.410310202

[78] Beal, M.F. (1992) Role of excitotoxicity in human neurological disease. Current Opinion in Neurobiology, 2, $657-$ 662. doi:10.1016/0959-4388(92)90035-J

[79] Liot, G., et al. (2009) Complex II inhibition by 3-NP causes mitochondrial fragmentation and neuronal cell death via an NMDA- and ROS-dependent pathway. Cell Death Differ, 16, 899-909. doi:10.1038/cdd.2009.22

[80] Schulz, J.B., et al. (1996) Involvement of oxidative stress in 3-nitropropionic acid neurotoxicity. Neurochemistry International, 29, 167-171. doi:10.1016/0197-0186(95)00122-0

[81] Schulz, J.B., et al. (1997) The role of mitochondrial dysfunction and neuronal nitric oxide in animal models of neurodegenerative diseases. Molecular and Cellular Biochemistry, 174, 193-197. doi:10.1023/A:1006852306789

[82] Sies, H. and Cadenas, E. (1985) Oxidative stress: Damage to intact cells and organs. Philosophical Transactions of the Royal Society B: Biological Sciences, 311, 617-631. doi:10.1098/rstb.1985.0168

[83] Vercesi, A.E., et al. (1997) The role of reactive oxygen species in mitochondrial permeability transition. Bioscience Reports, 17, 43-52. doi:10.1023/A:1027335217774

[84] Beal, M.F., et al. (1995) 3-Nitropropionic acid neurotoxicity is attenuated in copper/zinc superoxide dismutase transgenic mice. Journal of Neurochemistry, 65, 919-922.

[85] Galpern, W.R., et al. (1996) NGF attenuates 3-nitrotyrosine formation in a 3-NP model of Huntington's disease. Neuroreport, 7, 2639-2642.

[86] Schulz, J.B., et al. (1995) Blockade of neuronal nitric oxide synthase protects against excitotoxicity in vivo. The Journal of Neuroscience, 15, 8419-8429.

[87] Browne, S.E., et al. (1997) Oxidative damage and metabolic dysfunction in Huntington's disease: Selective vulnerability of the basal ganglia. Annals of Neurology, 41, 646-653. doi:10.1002/ana.410410514

[88] Good, P.F., et al. (1998) Protein nitration in Parkinson's disease. Journal of Neuropathology \& Experimental Neurology, 57, 338-342. doi:10.1097/00005072-199804000-00006

[89] Hensley, K., et al. (1998) Electrochemical analysis of protein nitrotyrosine and dityrosine in the Alzheimer brain indicates region-specific accumulation. The Journal of Neuroscience, 18, 8126-8132.

[90] Hanafy, K.A., Krumenacker, J.S. and Murad, F. (2001) $\mathrm{NO}$, nitrotyrosine, and cyclic GMP in signal transduction. Medical Science Monitor, 7, 801-819.

[91] Kong, S.K., et al. (1996) Peroxynitrite disables the tyrosine phosphorylation regulatory mechanism: Lymphocyte-specific tyrosine kinase fails to phosphorylate nitrated cdc2(6-20) $\mathrm{NH}_{2}$ peptide. Proceedings of the National Academy of Sciences of United States, 93, 3377-3382. doi:10.1073/pnas.93.8.3377

[92] Stadtman, E.R. (2001) Protein oxidation in aging and agerelated diseases. Annals of the New York Academy of Sciences, 928, 22-38.

[93] Huie, R.E. and Padmaja, S. (1993) The reaction of no 
with superoxide. Free Radical Research Communications, 18, 195-199. doi:10.3109/10715769309145868

[94] Esposito, L.A., et al. (1999) Mitochondrial disease in mouse results in increased oxidative stress. Proceedings of the National Academy of Sciences of United States, 96, 4820-4825. doi:10.1073/pnas.96.9.4820

[95] Geddes, J.W. and Pang, Z. (2000) Mechanisms of 3-nitropropionic acid toxicity. In: Sanberg, P.R., Nishino, H. and Borlongan, C.V., Eds., Mitochondrial Inhibitors and Neurodegenerative Disorders. Humana Press, Totowa, 107-120. doi:10.1007/978-1-59259-692-8 7

[96] Kaufmann, S.H., et al. (1993) Specific proteolytic cleavage of poly (ADP-ribose) polymerase: An early marker of chemotherapy-induced apoptosis. Cancer Research, 53, 3976-3985.

[97] Lazebnik, Y.A., et al. (1994) Cleavage of poly (ADPribose) polymerase by a proteinase with properties like ICE. Nature, 371, 346-347. doi:10.1038/371346a0

[98] Kerr, J.F. (2002) History of the events leading to the formulation of the apoptosis concept. Toxicology, 181-182, 471-474. doi:10.1016/S0300-483X(02)00457-2

[99] Hamilton, B.F. and Gould, D.H. (1987) Nature and distribution of brain lesions in rats intoxicated with 3-nitropropionic acid: A type of hypoxic (energy deficient) brain. Acta Neuropathologica (Berlin), 72, 286-297. doi: 10.1007/BF00691103

[100] Miller, P.J. and Zaborszky, L. (1997) 3-Nitropropionic acid neurotoxicity: Visualization by silver staining and implications for use as an animal model of Huntington's disease. Experimental Neurology, 146, 212-229. doi:10.1006/exnr.1997.6522

[101] Brinkhurst, F.R. and Potts, Jr., J.T. (1979) Calcium and phosphate distribution, turnover, and metabolic actions. Endocrinology, 2, 551-585.

[102] Kass, G.E. and Orrenius, S. (1999) Calcium signaling and cytotoxicity. Environmental Health Perspectives, 107, 2535 .

[103] Cheung, W.Y. (1982) Calmodulin: An overview. Federation Proceedings, 41, 2253-2257.

[104] Rizzuto, R., et al. (1994) Mitochondrial $\mathrm{Ca}^{2+}$ homeostasis in intact cells. The Journal of Cell Biology, 126, 11831194. doi: $10.1083 / \mathrm{jcb} .126 .5 .1183$

[105] Rutter, G.A., et al. (1996) Subcellular imaging of intramitochondrial $\mathrm{Ca}^{2+}$ with recombinant targeted aequorin: Significance for the regulation of pyruvate dehydrogenase activity. Proceedings of the National Academy of Sciences of United States, 93, 5489-5494. doi:10.1073/pnas.93.11.5489

[106] Bernardi, P. (1999) Mitochondrial transport of cations: Channels, exchangers, and permeability transition. Physiological Reviews, 79, 1127-1155.

[107] Nicholls, D.G. (2004) Mitochondrial dysfunction and glutamate excitotoxicity studied in primary neuronal cultures. Current Molecular Medicine, 4, 149-177. doi:10.2174/1566524043479239

[108] Jou, M.J., et al. (2004) Mitochondrial reactive oxygen species generation and calcium increase induced by visible light in astrocytes. Annals of the New York Academy of Sciences, 1011, 45-56.

doi:10.1196/annals.1293.005

[109] Jou, M.J., et al. (2010) Visualization of melatonin's multiple mitochondrial levels of protection against mitochondrial $\left.\mathrm{Ca}^{2+}\right)$-mediated permeability transition and beyond in rat brain astrocytes. Journal of Pineal Research, 48, 20-38. doi:10.1111/j.1600-079X.2009.00721.x

[110] Peng, T.I., et al. (2005) Mitochondrion-targeted photosensitizer enhances the photodynamic effect-induced mitochondrial dysfunction and apoptosis. Annals of the New York Academy of Sciences, 1042, 419-428. doi:10.1196/annals.1338.035

[111] Peng, T.I. and Jou, M.J. (2004) Mitochondrial swelling and generation of reactive oxygen species induced by photoirradiation are heterogeneously distributed. Annals of the New York Academy of Sciences, 1011, 112-122. doi:10.1196/annals.1293.012

[112] Sato, T. and Tauchi, H. (1982) Age changes of mitochondria of rat kidney. Mechanisms of Ageing and Development, 20, 111-126. doi:10.1016/0047-6374(82)90063-X

[113] Mecocci, P., MacGarvey, U. and Beal, M.F. (1994) Oxidative damage to mitochondrial DNA is increased in Alzheimer's disease. Annals of Neurology, 36, 747-751. doi:10.1002/ana.410360510

[114] Kim, G.W. and Chan, P.H. (2001) Oxidative stress and neuronal DNA fragmentation mediate age-dependent vulnerability to the mitochondrial toxin, 3-nitropropionic acid, in the mouse striatum. Neurobiology of Disease, 8, 114-126. doi:10.1006/nbdi.2000.0327

[115] Sohal, R.S. and Weindruch, R. (1996) Oxidative stress, caloric restriction, and aging. Science, 273, 59-63. doi:10.1126/science.273.5271.59

[116] Beal, M.F., et al. (1993) Neurochemical and histologic characterization of striatal excitotoxic lesions produced by the mitochondrial toxin 3-nitropropionic acid. The Journal of Neuroscience, 13, 4181-4192.

[117] Brouillet, E. and Hantraye, P. (1995) Effects of chronic MPTP and 3-nitropropionic acid in nonhuman primates. Current Opinion in Neurology, 8, 469-473. doi:10.1097/00019052-199512000-00014

[118] Nishino, H., et al. (1995) Chronically administered 3nitropropionic acid induces striatal lesions attributed to dysfunction of the blood-brain barrier. Neuroscience Letters, 186, 161-164. doi:10.1016/0304-3940(95)11311-J

[119] Nishino, H., et al. (2000) The striatum is the most vulnerable region in the brain to mitochondrial energy compromise: a hypothesis to explain its specific vulnerability. Journal of Neurotrauma, 17, 251-260. doi:10.1089/neu.2000.17.251

[120] Brustovetsky, N. and Dubinsky, J.M. (2000) Dual responses of CNS mitochondria to elevated calcium. The Journal of Neuroscience, 20, 103-113.

[121] Brustovetsky, N., et al. (2003) Increased susceptibility of striatal mitochondria to calcium-induced permeability transition. The Journal of Neuroscience, 23, 4858-4867.

[122] Basso, E., et al. (2005) Properties of the permeability tran- 
sition pore in mitochondria devoid of Cyclophilin D. The Journal of Biological Chemistry, 280, 18558-18561. doi:10.1074/jbc.C500089200

[123] Wullner, U., et al. (1994) 3-Nitropropionic acid toxicity in the striatum. Journal of Neurochemistry, 63, 17721781. doi:10.1046/j.1471-4159.1994.63051772.x

[124] Fu, Y., et al. (1995) 3-Nitropropionic acid produces indirect excitotoxic damage to rat striatum. Neurotoxicology and Teratology, 17, 333-339. doi:10.1016/0892-0362(94)00076-P

[125] Shimano, Y., et al. (1995) Chronically administered 3-nitropropionic acid produces selective lesions in the striatum and reduces muscle tonus. Obesity Research, 3, S779-S784. doi:10.1002/j.1550-8528.1995.tb00499.x

[126] Reynolds, D.S., Carter, R.J. and Morton, A.J. (1998) Dopamine modulates the susceptibility of striatal neurons to 3-nitropropionic acid in the rat model of Huntington's disease. The Journal of Neuroscience, 18, 10116-10127.

[127] Pandey, M., et al. (2009) Striatal dopamine level contributes to hydroxyl radical generation and subsequent neurodegeneration in the striatum in 3-nitropropionic acidinduced Huntington's disease in rats. Neurochemistry In- ternational, 55, 431-437. doi:10.1016/j.neuint.2009.04.013

[128] Villaran, R.F., et al. (2008) Endogenous dopamine enhances the neurotoxicity of 3-nitropropionic acid in the striatum through the increase of mitochondrial respiratory inhibition and free radicals production. Neurotoxicology, 29, 244-258.

[129] Lindal, S. (2002) Mitochondria and neurodegenerative diseases, is there a link? The role of mitochondria in the pathogenesis of amyotrophic lateral sclerosis (ALS). Ultrastructural Pathology, 26, 1-2. doi:10.1080/01913120252934251

[130] Horton, T.M., et al. (1995) Marked increase in mitochondrial DNA deletion levels in the cerebral cortex of Huntington's disease patients. Neurology, 45, 1879-1883. doi:10.1212/WNL.45.10.1879

[131] Hu, T. and Desai, J.P. (2004) Soft-tissue material properties under large deformation: Strain rate effect. Proceedings of the 26th Annual International Conference of the IEEE EMBS, San Francisco, 1-5 September 2004, 27582761 . 